\title{
RAMBA: Wearable Pneumatic Leg Compression Device
}

\author{
J Bamalakshmi ${ }^{1}, \mathrm{~K}$ Renuka ${ }^{2}$ \\ Pondicherry Journal of Nursing (2019): 10.5005/jp-journals-10084-12116
}

\section{Statement of Invention}

The present device is RAMBA:Wearable Pneumatic Leg Compression Device, which can be used for high-risk hospitalized clients (low grade)/nonhospitalized high-risk groups (general public) in order to prevent the occurrence of deep vein thrombosis (DVT).

Deep vein thrombosis is important to know because it can happen to anybody and can cause serious illness, disability, and, in some cases, death. Certain factors and situations can increase the risk of developing potentially deadly blood clots around $86 \%$ among hospitalized patients undergone any surgical therapy and prolonged bedridden patients, 63\% among nonhospitalized clients such as people who stand all day at work, 39\% among chronic smokers, and $76 \%$ among obese. ${ }^{1}$ Prevention is better than cure so by providing appropriate measures to high risk for DVT among hospitalized and non hospitalized clients to prevent the occurrence of DVT. ${ }^{2}$ Pneumatic compressions have been shown to be effective in introducing postoperative venous thrombosis. Hospital-acquired DVT and pulmonary embolisms (PE) are preventable problems that can increase mortality. ${ }^{3}$ RAMBA: Wearable Pneumatic Leg Compression Device is used for high-risk hospitalized clients (low grade)/nonhospitalized high-risk groups (general public) in order to prevent the occurrence of DVT. Pneumatic leg compression device available in India gives sequential compression and relaxation on the vein, which helps in preventing occurrence of DVT among high-risk clients. But the drawback of the existing pneumatic leg compression device is that the patients will have restricted movement and will be confined to the bed. So, we have developed a new wearable pneumatic leg compression device that has only one air compartment for compression and relaxation with fixed pressure of $80 \mathrm{~mm} \mathrm{Hg}$. Timing will be fixed as 18 seconds for compression and 12 seconds for relaxation at the rate of two cycles in 1 minute and the client need not be restricted to the bed as well as they can do normal day-to-day activities. High-risk groups for DVT are security guards, nurses, teachers, tailors, smokers, old-age people, obese, pregnant women, bus drivers, and conductors. These high-risk groups can not be protected from occurrence of DVT by the existing product. RAMBA: wearable pneumatic leg compression device can be applied every 3 month once up to 15 days among nonhospitalized high-risk groups (general public) in order to prevent the occurrence of DVT.

\section{Description of the Present Invention}

The present invention is RAMBA: wearable pneumatic leg compression device, which can be used as a portable base so that the client can do normal activity. The cuff will be worn on the calf muscles and it will inflate and deflate digitally using the compressor motor and the solenoid valve, respectively, to give compression and relaxation effects. A software is fixed for program control. The pressure sensor is to control pressure duration in
${ }^{1}$ Department of Community Health Nursing, Kasturba Gandhi Nursing College, Sri Balaji Vidyapeeth Deemed University, Puducherry, India

${ }^{2}$ Department of Medical Surgical Nursing, Kasturba Gandhi Nursing College, Sri Balaji Vidyapeeth Deemed University, Puducherry, India

Corresponding Author: J Bamalakshmi, Department of Community Health Nursing, Kasturba Gandhi Nursing College, Sri Balaji Vidyapeeth Deemed University, Puducherry, India, Phone: +91 9659366979, e-mail: vinoth.bsf@gmail.com

How to cite this article: Bamalakshmi J, Renuka K. RAMBA: Wearable Pneumatic Leg Compression Device. Pon J Nurs 2019;12(2):52-53.

Source of support: Sri Balaji Vidyapeeth University

Conflict of interest: None

cycle. Cuff size will be $15 \mathrm{~cm}$ in breadth and $35 \mathrm{~cm}$ in length (Fig. 1). Pressure is fixed at maximum $80 \mathrm{~mm} \mathrm{Hg}$ to produce desirable effects (Fig. 2).

When the switch is $\mathrm{ON}$, automatically compression and relaxation will occur as per preset time, cycle, and pressure.

\section{How to USE?}

The cuff is tied to both legs below the knee in calf muscles, and device is switched ON. Automatic inflation and deflation occur with prefixed pressure and timings. Display should be monitored and the device should be used for 1 hour everyday up to 15 days.

\section{Advantages of the Present Device}

\section{- Light weight}

- Able to do normal activity after wearing the device

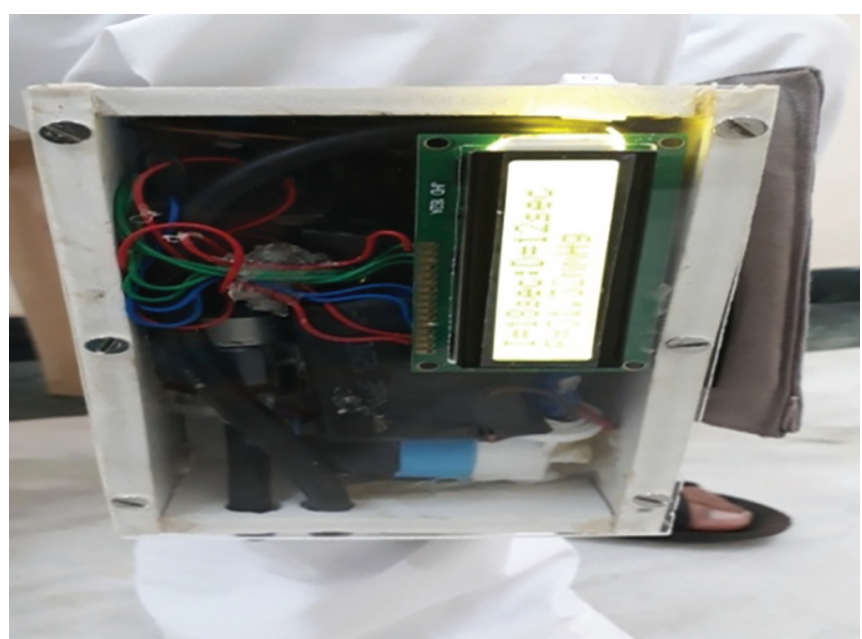

Fig. 1: RAMBA-Wearable pneumatic leg compression device

o The Author(s). 2019 Open Access This article is distributed under the terms of the Creative Commons Attribution 4.0 International License (https://creativecommons. org/licenses/by-nc/4.0/), which permits unrestricted use, distribution, and non-commercial reproduction in any medium, provided you give appropriate credit to the original author(s) and the source, provide a link to the Creative Commons license, and indicate if changes were made. The Creative Commons Public Domain Dedication waiver (http://creativecommons.org/publicdomain/zero/1.0/) applies to the data made available in this article, unless otherwise stated. 


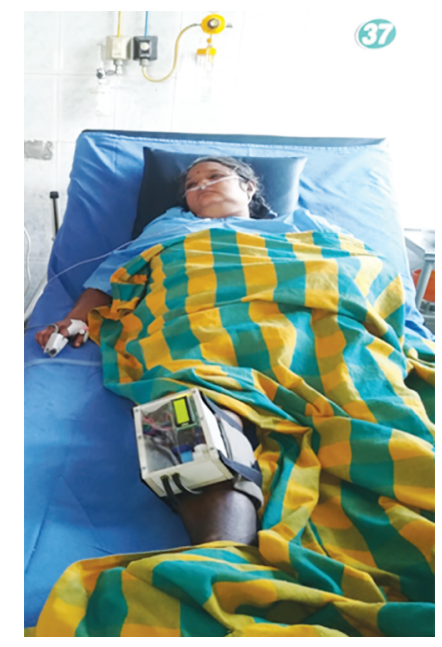

Fig. 2: Applied RAMBA: wearable pneumatic leg compression device to hospitalized client

- Can be used even if no electricity is available

- Easy to carry

- No need of heavy air compressor

- Air can be filled by mechanical air cuff bags

- Cost-effective ${ }^{4}$

\section{Benefits}

- Helps in increasing blood flow through the veins

- Prevents blood clots in the deep vein of the legs

- Pain and discomfort relief from reducing swelling in the legs

\section{Indication (High Risk for DVT)}

- Inheriting a blood clotting disorder

- Prolonged bedrest such as during a long hospital stay or paralysis

- Birth-control pills or hormone replacement therapy

- Chronic hypertension

- Malignancy

- Obesity

- Immobility
- Recent injury

- Chronic smokers

- Stroke

- Paralysis

- Chronic diabetes mellitus

- People with or at risk of circulatory problems like DVT and varicose vein

- Those who are chronic bedridden or have a hard time moving their legs

- People who stand all day at work

- Athletes

- Pregnant women ${ }^{5}$

\section{Contraindications}

- Severe arteriosclerosis or ischemic diseases

- Acute or severe DVT

- Severe congestive cardiac failure

- Pulmonary embolism or thrombophlebitis ${ }^{5}$

\section{Conclusion}

The present invention shall disclose a portable, light-weight, costeffective, wearable pneumatic leg compression device RAMBA that does not demand restriction to bed for high-risk people to treat/prevent the occurrence of DVT by preventing the venous thromboembolism in hospitalized patients and nonhospitalized high-risk people. RAMBA - pneumatic leg compression device is a multimodality approach to high-risk clients for DVT and is strongly recommended.

\section{References}

1. Galson SK. The surgeon general's call to action to prevent deep vein thrombosis and pulmonary embolism. Medical Surgical 2013;1:32.

2. Linda S. Willianms/Paula D. Hopper "Medical Surgical Nursing". 5th ed., Jaypee Brothers Medical Publishers (P) Ltd; 2015. p. 475.

3. Lewis SL, Mani CM. Lewis's Medical Surgical Nursing. Elsevier; 2011. p. 897.

4. Phillips N. Berry \& Kohn's Operative Room Technique. 11th ed., Mosby; 2007. p. 551.

5. Phipps W, Monahan F, Sands JK, Marek JF, Neighbors M. Medical Surgical Nursing. 7th ed., Mosby; 2003. p. 795. 